\title{
On the number of possible row and column sums of 0,1-matrices
}

\author{
Daniel Goldstein and Richard Stong \\ Center for Communications Research \\ 4320 Westerra Court \\ San Diego, CA 92121 \\ dgoldste@ccrwest.org \\ Department of Mathematics \\ Rice University \\ Houston, TX 77005 \\ stong@math.rice.edu
}

Submitted: Aug 9, 2005; Accepted: Apr 4, 2006; Published: Apr 18, 2006

Mathematics Subject Classification: 05A15

\begin{abstract}
For $n$ a positive integer, we show that the number of of $2 n$-tuples of integers that are the row and column sums of some $n \times n$ matrix with entries in $\{0,1\}$ is evenly divisible by $n+1$. This confirms a conjecture of Benton, Snow, and Wallach.

We also consider a $q$-analogue for $m \times n$ matrices. We give an efficient recursion formula for this analogue. We prove a divisibility result in this context that implies the $n+1$ divisibility result.
\end{abstract}

\section{Introduction}

We study the number $p(m, n)$ of $(m+n)$-tuples of integers that are the row and column sums of some $m \times n$ matrix with entries in $\{0,1\}$. For each $n \geq 1$, the sequence $\{p(m, n)\}_{m=1}^{\infty}$ is a linear recursion of degree $n$. Moreover, this recursion is annihilated by the polynomial $(T-(n+1))^{n}$. It follows that if $1 \leq n \leq m$, then $p(m, n)$ is evenly divisible by $(n+1)^{m-n+1}$. This confirms a conjecture of Benton, Snow, and Wallach.

For positive integers $m$ and $n$, let $\mathcal{M}=\mathcal{M}_{m, n}$ be the set of $m \times n$ matrices with entries in $\{0,1\}$. For $M$ in $\mathcal{M}$, we write $M=\left(M_{i j}\right)$.

We have two vector-valued functions on $\mathcal{M}$ : the vector $x(M)=\left(x_{1}, \ldots, x_{m}\right)$ of row sums, where $x_{i}=\sum_{1 \leq j \leq n} M_{i j}$ for $1 \leq i \leq m$, and the vector $y(M)=\left(y_{1}, \ldots, y_{n}\right)$ of column sums, where $y_{j}=\sum_{1 \leq i \leq m} M_{i j}$ for $1 \leq j \leq n$.

Define $\mathcal{R C}=\mathcal{R C}_{m, n}$ to be the set of pairs of row and column sums $(x(M), y(M))$ as $M$ ranges over $\mathcal{M}$. Our main result concerns the cardinality $p(m, n)$ of $\mathcal{R C}_{m, n}$. 
Theorem 1 We have

1. $p(1,1)=2$.

2. $p(m, n)=p(n, m)$ for $m, n \geq 1$.

3. If $1 \leq n \leq m$, then $p(m, n)=\sum_{1 \leq i \leq n}(-1)^{i+1}\left(\begin{array}{c}n \\ i\end{array}\right)(n+1)^{i} p(m-i, n)$.

Of these statements, part (1) is clear, and part (2) follows by taking transpose, for $x\left(M^{t}\right)=y(M)$ and $y\left(M^{t}\right)=x(M)$.

Part (3) says that, for each $n \geq 1$, the sequence $\{p(m, n)\}_{m=1}^{\infty}$ is a linear recursion of degree $n$ that is annihilated by the polynomial $(T-(n+1))^{n}$. Note that, for any fixed $n$, the recursion (3) is equivalent to $p(m, n)=r_{n}(m)(n+1)^{m}$ for some polynomial $r_{n}(m)$ of degree $\leq n-1$.

Part (3) implies the following corollary.

Corollary 2 The number $p(m, n)$ is evenly divisible by $(n+1)^{m-n+1}$ if $1 \leq n \leq m$.

Indeed each of the $n$ terms in the sum representing $p(m, n)$ is divisible by this quantity. A second consequence of part (3) is an efficient algorithm for computing $p(m, n)$.

Algorithm 3 We construct a table of the values $p(i, j)$, for $1 \leq i, j \leq m$ by induction on $j$. First we fill in $p(i, 1)=2^{i}$, for $1 \leq i \leq m$. Next, for a given $j \leq m$, having filled in $p\left(i, j^{\prime}\right)$ for $1 \leq j^{\prime}<j$, we fill in $p(i, j)$ by induction on $i$, using part (2) if $i \leq j$ and part (3) if $i>j$.

\section{A generalization}

We mention a mild generalization of Theorem 1 and its corollary. Define the polynomial $P=P_{m, n}(q)=\sum_{(x, y) \in \mathcal{R C}_{m, n}} q^{|x|}$, where $|x|=x_{1}+\cdots+x_{m}$. We recover $p(m, n)$ by evaluating the polynomial $P_{m, n}$ at $q=1$.

Theorem 4 We have

1. $P_{1,1}=1+q$.

2. $P_{m, n}=P_{n, m}$ for $m, n \geq 1$.

3. If $1 \leq n \leq m$, then $P_{m, n}=\sum_{1 \leq i \leq n}(-1)^{i+1}\left(\begin{array}{c}n \\ i\end{array}\right)\left(1+q+\cdots+q^{n}\right)^{i} P_{m-i, n}$.

4. If $1 \leq n \leq m$, then the polynomial $P_{m, n}$ is evenly divisible by $\left(1+q+\cdots+q^{n}\right)^{m-n+1}$ in $\mathbb{Z}[x]$.

Part (4) answers a conjecture of J. Benton, R. Snow, and N. Wallach in [1]. 


\section{$3 \quad$ Start of the proof}

Let $\mathbb{N}=\{0,1, \ldots\}$. Define the weight of a matrix $N$ to be the sum of its entries, and write $|N|$ for the weight of $N$. With this definition, we have $|x(M)|=|M|=|y(M)|$ for $M \in \mathcal{M}$. Thus, a necessary condition for $x$ and $y$ to be row and column sums of a matrix is that they have the same weight.

Clearly, the row sums of a member of $\mathcal{M}$ are at most $n$. Conversely, if $x=\left(x_{1}, \ldots, x_{m}\right)$ and $0 \leq x_{i} \leq n$, let $R=R(x)$ be the $m \times n$ matrix such that $R_{i j}=1$ if $1 \leq j \leq x_{i}$ and $R_{i j}=0$ otherwise. Then $R$ lies in $\mathcal{M}$ and has row sums equal to $x$. This proves:

Lemma 5 Let $x=\left(x_{1}, \ldots, x_{m}\right) \in \mathbb{N}^{m}$. Then $x$ is the vector of row sums of an $m \times n$ matrix with entries in $\{0,1\}$ if and only if $x_{i} \leq n$ for all $i$.

Let $a_{j}$ be the number of rows of $R$ that have exactly $j$ ones. Write $a=\left(a_{0}, \ldots, a_{n}\right)=$ $a(x)$ in $\mathbb{N}^{n+1}$. We note that $|a|=m$, and write $\left(\begin{array}{c}m \\ a\end{array}\right)$ for the multinomial coefficient $\frac{m !}{a_{0} ! \cdots a_{n} !}$ With this notation, we have the following lemma.

Lemma 6 Let a in $\mathbb{N}^{n+1}$ satisfy $|a|=m$. Then the number of $x$ in $\mathbb{N}^{m}$ such that $a(x)=a$ is $\left(\begin{array}{c}m \\ a\end{array}\right)$.

Let $\lambda=\left(\lambda_{1}, \ldots, \lambda_{n}\right)=\lambda(x)$ be the column sums of the matrix $R$ constructed above. It satisfies the dominance condition:

$$
\lambda_{1} \geq \cdots \geq \lambda_{n}
$$

Note that $a$ in $\mathbb{N}^{n+1}$ with $|a|=m$ determines a dominant $\lambda$ in $\mathbb{N}^{n}$ with $m \geq \lambda_{1}$, and vice versa. For, given $\lambda$, set $\lambda_{0}=m$ and $\lambda_{n+1}=0$, and define $a_{j}=\lambda_{j}-\lambda_{j+1}$, for $j=0, \ldots, n$. Conversely, given $a$ in $\mathbb{N}^{n+1}$, define $\lambda_{j}=a_{j}+\cdots+a_{n}$.

The weights of these vectors are related by $|x|=|\lambda|=\sum_{0 \leq j \leq n} j a_{j}$.

Given $y, \lambda$ in $\mathbb{N}^{n}$ with $\lambda$ dominant, we define $y \preceq \lambda$ if

$$
y_{1}+\cdots+y_{j} \leq \lambda_{1}+\cdots+\lambda_{j}
$$

for all $j$ in the range $1 \leq j \leq n$.

The symmetric group $S_{n}$ acts on $\mathbb{N}^{n}$ by permuting coordinates. For $y \in N^{n}$ and $\sigma \in S_{n}$, we set $y \sigma=\left(y_{\sigma(1)}, \ldots, y_{\sigma(n)}\right)$.

The next result, proved in [2, Corollary 6.2.5] or [3, Theorem 16.1], gives necessary and sufficient conditions for a pair of vectors to lie in $\mathcal{R C}_{m, n}$.

Lemma 7 Let $x$ in $\mathbb{N}^{m}$ be the vector of row sums of a matrix in $\mathcal{M}$, and set $\lambda=\lambda(x)$. Then $(x, y) \in \mathcal{R C}$ if and only if $y \in \mathbb{N}^{n}$ satisfies

(i) $|y|=|\lambda|$, and

(ii) $y \sigma \preceq \lambda$ for all $\sigma \in S_{n}$. 
Let $N(\lambda)$ be the number of $y \in \mathbb{N}^{n}$ that satisfy (i) and (ii). Then

$$
P_{m, n}(q)=\sum_{x \in\{0, \ldots, n\}^{m}} N(\lambda(x)) q^{|x|} .
$$

Combined with Lemma 6, this gives:

$$
P_{m, n}(q)=\sum_{\substack{a \in \mathbb{N}^{n+1} \\
|a|=m}}\left(\begin{array}{c}
m \\
a
\end{array}\right) N(\lambda) q^{a_{1}+2 a_{2}+\cdots+n a_{n}}
$$

\section{Key Lemma}

Lemma 8 Let $n \geq 1$. There is a polynomial $G=G_{n}$ in $\mathbb{Q}\left[z_{1}, \ldots, z_{n}\right]$ of total degree $\leq n-1$ such that $N(\lambda)=G\left(\lambda_{1}, \ldots, \lambda_{n}\right)$ for any dominant $\lambda=\left(\lambda_{1}, \ldots, \lambda_{n}\right)$ in $\mathbb{N}^{n}$.

To count $N(\lambda)$, we will condition on the first term $y_{1}$ of the vector $y$. We will need a subsidiary function. Let $N(\lambda ; t)$ be the number of solutions of (i) and (ii) with $y_{1}=t$. By definition, $N(\lambda)=\sum_{t \geq 0} N(\lambda ; t)$.

We need one more definition to state the next lemma. Suppose $\lambda=\left(\lambda_{1}, \ldots, \lambda_{n}\right)$ has $n$ parts, and $\lambda_{j+1}<t \leq \lambda_{j}$. Then we define $\mu(t)$ with $n-1$ parts to be

$$
\mu(t)=\left(\lambda_{1}, \ldots, \lambda_{j-1}, \lambda_{j}+\lambda_{j+1}-t, \lambda_{j+2}, \ldots, \lambda_{n}\right) .
$$

(In the definition of $\mu(t), \lambda_{j}$ and $\lambda_{j+1}$ have been removed and $\lambda_{j}+\lambda_{j+1}-t$ has been inserted.) Note that if $\lambda$ is dominant, then so also is $\mu(t)$ since $\lambda_{j}>\lambda_{j}+\lambda_{j+1}-t \geq \lambda_{j+1}$.

Lemma 9 We have:

(a) If $t<\lambda_{n}$ or if $t>\lambda_{1}$, then $N(\lambda ; t)=0$.

(b) $N\left(\lambda ; \lambda_{n}\right)=N\left(\left(\lambda_{1}, \ldots, \lambda_{n-1}\right)\right)$.

(c) Suppose that $\lambda_{j+1}<t \leq \lambda_{j}$. Then $N(\lambda ; t)=N(\mu(t))$.

Proof. If $y_{1}>\lambda_{1}$ then (ii) is violated. Suppose $y$ satisfies (i) and $y_{1}<\lambda_{n}$. Then

$$
y_{2}+y_{3}+\cdots+y_{n}>\lambda_{1}+\lambda_{2}+\cdots+\lambda_{n-1},
$$

thus (ii) is violated if $\sigma(n)=1$. Therefore $N\left(\lambda, y_{1}\right)=0$, proving (a), and we turn to (b).

Set $\lambda^{\prime}=\left(\lambda_{1}, \ldots, \lambda_{n-1}\right)$. We claim that the correspondence

$$
\left(y_{1}, y_{2} \ldots, y_{n}\right) \longleftrightarrow\left(y_{2} \ldots, y_{n}\right)
$$

gives a bijection between the sets counting $N\left(\lambda ; y_{1}\right)$ and $N\left(\lambda^{\prime}\right)$. One direction follows by definition: if $\left(y_{1}, \ldots, y_{n}\right)$ is counted by $N(\lambda)$, then $\left(y_{2}, \ldots, y_{n}\right)$ is counted by $N\left(\lambda^{\prime}\right)$. 
Conversely, suppose that $\left(y_{2}, \ldots, y_{n}\right)$ is counted by $N\left(\lambda^{\prime}\right)$. Now (i) (for $y$ and $\lambda$ ) follows since $y_{1}=\lambda_{n}$. To prove (ii), let $\sigma \in S_{n}$. Set $k=\sigma^{-1}(1)$. Now

$$
\begin{aligned}
y_{\sigma(1)}+\cdots+y_{\sigma(j)} & \leq\left(\lambda_{1}+\cdots+\lambda_{j-1}\right)+\lambda_{n} \\
& \leq \lambda_{1}+\cdots+\lambda_{j}
\end{aligned}
$$

if $j \geq k$. The inequality is clear if $j<k$.

Part (c) is proved using the same correspondence used in part (b). The straightforward but tedious calculation is omitted.

Proof of Lemma 8. Suppose $n=1$ and let $\lambda=\left(\lambda_{1}\right)$. Then $N\left(\lambda_{1}\right)=1$, a polynomial of degree 0 .

Thus the lemma holds for $n=1$. We proceed by induction to prove it for all $n$. Suppose the lemma has been proved for $n$ and we wish to prove it for $n+1$.

We break up the sum that counts $N(\lambda)$, by conditioning on $y_{1}$. By Lemma 9 (a), it is enough to consider $y_{1}$ in the range $\lambda_{n} \leq y_{1} \leq \lambda_{1}$. Either $y_{1}=\lambda_{n}$, or $\lambda_{j+1}<y_{1} \leq \lambda_{j}$ for a unique $j$ in the range $1 \leq j<n$, and therefore

$$
N(\lambda)=N\left(\lambda ; \lambda_{n}\right)+\sum_{1 \leq j<n} \sum_{\lambda_{j+1}<t \leq \lambda_{j}} N(\lambda ; t) .
$$

In view of Lemma 9(b) and (c), this yields

$$
N(\lambda)=N\left(\left(\lambda_{1}, \ldots, \lambda_{n-1}\right)\right)+\sum_{1 \leq j<n} \sum_{\lambda_{j+1}<t \leq \lambda_{j}} N(\mu(t))
$$

To see that $N(\lambda)$ is a polynomial of degree at most $n$, it suffices to show that each term on the right is a polynomial of total degree at most $n$. This is true for the first term $N\left(\left(\lambda_{1}, \ldots, \lambda_{n-1}\right)\right)$ by the inductive hypothesis.

Each of the subsequent terms is itself a sum. By the inductive hypothesis, each summand in each term is a polynomial of degree $\leq n-1$. But, for any polynomial $f$, we have that $\sum_{x<t \leq y} f(t)$ is a polynomial in $x$ and $y$ of degree $\leq \operatorname{deg} f+1$.

By induction and (4) it follows that the coefficients of $G$ are rational numbers. This proves the lemma.

\section{End of the proof}

Since $G$ is a polynomial of degree $\leq n-1$ by Lemma 8, so also is $H$ defined by $H\left(a_{0}, a_{1}, \ldots, a_{n}\right)=G_{n}\left(\lambda_{1}, \ldots, \lambda_{n}\right)$, since the transformation from $\lambda$ to $a$ is linear.

By (3) we have

$$
P_{m, n}=\sum_{\substack{a \in \mathbb{N}^{n+1} \\
|a|=m}}\left(\begin{array}{c}
m \\
a
\end{array}\right) H\left(a_{0}, \ldots, a_{n}\right) q^{a_{1}+\cdots+n a_{n}}
$$


Proof of Theorem 4. We are free to assume $n \leq m$.

We define the function $E$ of the variables $z_{0}, \ldots, z_{n}$ by

$$
E\left(z_{0}, \ldots, z_{n}\right)=\sum_{\substack{a \in N^{n+1} \\
|a|=m}}\left(\begin{array}{c}
m \\
a
\end{array}\right) H\left(a_{0}, \ldots, a_{n}\right) e^{a_{0} z_{0}+\cdots+a_{n} z_{n}} .
$$

By (5) and (6), we have $P_{m, n}(q)=E(0, \log (q), 2 \log (q), \ldots, n \log (q))$.

The following lemma is proved by induction.

Lemma 10 Let $H \in \mathbb{Q}\left[z_{0}, \ldots, z_{n}\right]$ be a polynomial. Write $z=\left(z_{0}, \ldots, z_{n}\right)$ and $a=$ $\left(a_{0}, \ldots, a_{n}\right)$, and set $a \cdot z=a_{0} z_{0}+\cdots+a_{n} z_{n}$. Then there is a linear differential operator $D$ in $z_{0}, \ldots, z_{n}$ such that $H(z) e^{a \cdot z}=D e^{a \cdot z}$. Moreover, $\operatorname{deg}(D)=\operatorname{deg}(H)$.

By the lemma, we have

$$
E(z)=\sum_{\substack{a \in N^{n+1} \\
|a|=m}}\left(\begin{array}{c}
m \\
a
\end{array}\right) D e^{a \cdot z}=D\left(\sum\left(\begin{array}{c}
m \\
a
\end{array}\right) e^{a \cdot z}\right) .
$$

By the multinomial theorem

$$
\sum_{\substack{a \in N^{n+1} \\
|a|=m}}\left(\begin{array}{c}
m \\
a
\end{array}\right) e^{a \cdot z}=\left(e^{z_{0}}+\cdots+e^{z_{n}}\right)^{m},
$$

whence $E$ is $\left(e^{z_{0}}+\cdots+e^{z_{n}}\right)^{m-n+1}$ times a polynomial $f_{1}\left(m, e^{z_{0}}, \ldots, e^{z_{n}}\right)$ whose degree in $m$ is $\leq n-1$.

Set $f(m, q)=f_{1}\left(m, 1, q, \ldots, q^{n}\right)$. When evaluated at $z_{i}=i \log (q), e^{z_{0}}+\cdots+e^{z_{n}}$ becomes $\left(1+q+\cdots+q^{n}\right)$, whence $P_{m, n}=f(m, q)\left(1+q+\cdots+q^{n}\right)^{m-n+1}$. Since $f(m, q)$ is a polynomial in $m$ of degree at most $n-1$, part (3) follows immediately.

Set $\pi=\left(1+q+\cdots+q^{n}\right)^{n-m+1}$. Finally, to prove part (4), it remains to show that, for each $m$, the coefficients of $f(m, q)$, as a polynomial in $q$, are integers.

One way to see this is to regard $f=P_{m, n} / \pi$ as a power series identity and formally equate coefficients of $q^{i}$, because $\pi$ is a polynomial in $q$ with constant term 1 . Theorem 4 is proved.

\section{References}

[1] J. Benton, R. Snow, and N. Wallach. A combinatorial problem associated with nonograms, Linear Algebra and its Applications, Volume 412, Issue 1, 1 January 2006, Pages 30-38.

[2] R. H. Brualdi and H. J. Ryser. Combinatorial matrix theory. Cambridge University Press, 1991.

[3] J. H. van Lint and R. M. Wilson. A course in combinatorics. Cambridge University Press, 1992. 